\title{
Weak optimal inverse problems of interval linear programming based on KKT conditions
}

\author{
LIU Xiao $^{1} \quad$ JIANG Tao ${ }^{1,2 *} \quad$ LI Hao-hao ${ }^{3}$
}

\begin{abstract}
In this paper, weak optimal inverse problems of interval linear programming (IvLP) are studied based on KKT conditions. Firstly, the problem is precisely defined. Specifically, by adjusting the minimum change of the current cost coefficient, a given weak solution can become optimal. Then, an equivalent characterization of weak optimal inverse IvLP problems is obtained. Finally, the problem is simplified without adjusting the cost coefficient of null variable.
\end{abstract}

\section{$\S 1 \quad$ Introduction}

The inverse problem in optimization, as a classical topic, has aroused considerable interest for a long time, see $[4,11,27]$. With the rapid development of science, inverse problems have been applied to some new fields [8,26]. For example, Finn et al. [6] explored how inverse optimal control can be used to learn behaviors from demonstrations and apply to torque control of high-dimensional robotic systems.

Interval linear programming (IvLP) [5, 10,13,15-18,21,24], which is one of significant problems in the area of optimization, has made great progress in recent decades. Despite this, some questions, such as weak optimal solutions of IvLP remain unsolved. To solve these problems, Steuer [25] introduced three algorithms for calculating weak optimal solutions of interval cost coefficient linear programming (LP). Hladík [9] proposed a polynomial time algorithm to verify weak optimal feasible solution. With the growing maturity of IvLP, researchers' attention has been extended from the initial optimal solutions and optimal problems to other directions, such

\footnotetext{
Received: 2020-11-26. Revised: 2021-05-17.

MR Subject Classification: 65G40.

Keywords: interval linear programming, inverse problems, KKT conditions, weak optimal solution.

Digital Object Identifier(DOI): https://doi.org/10.1007/s11766-021-4324-2.

Supported by the National Natural Science Foundation of China(11971433), First Class Discipline of Zhejiang - A (Zhejiang Gongshang University- Statistics, 1020JYN4120004G-091), Graduate Scientific Research and Innovation Foundation of Zhejiang Gongshang University.

*Corresponding author.

(C)The Author(s) 2021.
} 
as the optimal value, multiobjective and inverse problems. Ishibuchi and Tanaka [12] established a method to transform the LP problem with interval cost coefficients into a multiobjective problem by introducing order relations. From the viewpoint of the order vector space, Li et al. [14] solved the admissible order multiobjective IvLP problem. Recently, Mohammadi and Gentili [20] proposed a new method to determine the optimal boundary.

Generally speaking, there are two types of inverse problems in optimization. One is the inverse optimal value problem [1], and the other is the inverse optimal solution problem [2]. Recently, Mostafaee et al. [19] have studied the inverse optimal value problem of IvLP. Through designing matrix games, the required optimal values could be achieved. The main idea is to determine that, under what circumstances, the specified optimal value can be obtained about the LP problem, in which the cost coefficient and the constraint coefficient are within the given interval. This provides a new direction for the research of IvLP. Based on this, we consider the second type of inverse problems, which is the inverse optimal solution problem of IvLP.

In this paper, we introduce the definition of weak optimal inverse IvLP problems, and present an equivalent characterization based on KKT conditions. In Section 2, the definition and conditions of weak optimal inverse IvLP problems are proposed, which can guide us in the search for a candidate of weak optimal inverse IvLP problems. In Section 3, an equivalent characterization of weak optimal inverse IvLP problems is obtained. Then, we simplify the problem without adjusting the cost coefficient of null variable and present the main result of this paper.

\section{$\S 2$ Preliminaries}

We first review some notations [5].

The set of all $m$-by- $n$ matrices and interval matrices will be denoted by $\mathbb{R}^{m \times n}$ and $\mathbb{I}^{m \times n}$ respectively. We define the interval matrix as

$$
\mathbf{A}=[\underline{A}, \bar{A}]=\left\{A \in \mathbb{R}^{m \times n}: \underline{A} \leq A \leq \bar{A}\right\} .
$$

The center and radius matrices of $\mathbf{A}$ are defined as

$$
A^{c}=\frac{1}{2}(\underline{A}+\bar{A}), A^{\Delta}=\frac{1}{2}(\bar{A}-\underline{A}),
$$

respectively. Then $\mathbf{A}=\left[A^{c}-A^{\Delta}, A^{c}+A^{\Delta}\right]$.

Denote the diagonal matrix $T_{y}=\operatorname{diag}\left(y_{1}, \ldots, y_{m}\right)$. Let $\{ \pm 1\}^{m}$ be the set of all $\{-1,1\}$ m-dimensional vectors, i.e. $\{ \pm 1\}^{m}=\left\{y \in \mathbb{R}^{m}|| y \mid=e\right\}$, where $e=(1, \cdots, 1)^{T}$ is the m-dimensional vector of all 1 's and the absolute value of a matrix $A=\left(a_{i j}\right)$ is defined by $|A|=\left(\left|a_{i j}\right|\right)$. Vector sign is denoted by

$$
(\operatorname{sign} x)_{i}= \begin{cases}1 & \text { if } x_{i} \geq 0, \\ -1 & \text { if } x_{i}<0 .\end{cases}
$$

Let $\mathbf{A} \in \mathbb{I}^{m_{1} \times n_{1}}, \mathbf{B} \in \mathbb{I}^{m_{1} \times n_{2}}, \mathbf{C} \in \mathbb{R}^{m_{2} \times n_{1}}, \mathbf{D} \in \mathbb{I}^{m_{2} \times n_{2}}, \mathbf{a} \in \mathbb{I}^{m_{1}}, \mathbf{b} \in \mathbb{R}^{m_{2}}, \mathbf{c} \in$ 
$\mathbb{I} \mathbb{R}^{1 \times n_{1}}$ and $\mathbf{d} \in \mathbb{R}^{1 \times n_{2}}$. We define the "forward" $\operatorname{IvLP}(\mathrm{FP})$ problem as

$$
\mathbf{F P}(\mathbf{c}, \mathbf{d}): \min \mathbf{c} x^{1}+\mathbf{d} x^{2} \text { s.t. } \mathbf{A} x^{1}+\mathbf{B} x^{2}=\mathbf{a}, \mathbf{C} x^{1}+\mathbf{D} x^{2} \leq \mathbf{b}, x^{1} \geq 0,
$$

which is the general IvLP problem.

The corresponding LP form of $\mathbf{F P}(\mathbf{c}, \mathbf{d})$ is stated as

$$
\mathbf{F P}(c, d): \min c x^{1}+d x^{2} \text { s.t. } A x^{1}+B x^{2}=a, C x^{1}+D x^{2} \leq b, x^{1} \geq 0 .
$$

Therefore, $\mathbf{F P}(\mathbf{c}, \mathbf{d})$ can be seemed as the set of $\mathbf{F P}(c, d)$ for any $A \in \mathbf{A}, B \in \mathbf{B}, C \in \mathbf{C}, D \in$ $\mathbf{D}, a \in \mathbf{a}, b \in \mathbf{b}, c \in \mathbf{c}, d \in \mathbf{d}$.

Definition 2.1. A vector $\bar{x}$ is called a weak solution to $\mathbf{F P}(\mathbf{c}, \mathbf{d})$, if for some $A \in \mathbf{A}, B \in$ $\mathbf{B}, C \in \mathbf{C}, D \in \mathbf{D}, a \in \mathbf{a}, b \in \mathbf{b}$, it satisfies

$$
A x^{1}+B x^{2}=a, C x^{1}+D x^{2} \leq b, x^{1} \geq 0 .
$$

Definition 2.2. A vector $\bar{x}$ is called a weak optimal solution to $\mathbf{F P}(\mathbf{c}, \mathbf{d})$, if for some $A \in$ $\mathbf{A}, B \in \mathbf{B}, C \in \mathbf{C}, D \in \mathbf{D}, a \in \mathbf{a}, b \in \mathbf{b}, c \in \mathbf{c}, d \in \mathbf{d}$, it is an optimal solution to $\mathbf{F P}(c, d)$.

Denote $\mathbf{X}^{W}(\mathbf{A}, \mathbf{B}, \mathbf{C}, \mathbf{D}, \mathbf{a}, \mathbf{b}), \mathbf{X}^{W O P}(\mathbf{c}, \mathbf{d})$ as the set of weak solutions and weak optimal solutions to $\mathbf{F P}(\mathbf{c}, \mathbf{d})$ respectively.

Let $\mathbf{X}(A, B, C, D, a, b), \mathbf{X}^{O P}(c, d)$ be the set of feasible solutions and optimal solutions to $\mathbf{F P}(c, d)$ respectively.

Assume that an observed solution $\bar{x} \in \mathbf{X}^{W}(\mathbf{A}, \mathbf{B}, \mathbf{C}, \mathbf{D}, \mathbf{a}, \mathbf{b}) \backslash \mathbf{X}^{W O P}(\mathbf{c}, \mathbf{d})$, i.e. for some $A \in \mathbf{A}, B \in \mathbf{B}, C \in \mathbf{C}, D \in \mathbf{D}, a \in \mathbf{a}, b \in \mathbf{b}, \bar{x} \in \mathbf{X}(A, B, C, D, a, b)$, but there is no $c \in \mathbf{c}, d \in \mathbf{d}$ such that $\bar{x} \in \mathbf{X}^{O P}(c, d)$.

Definition 2.3. For a given solution $\bar{x} \in \mathbf{X}^{W}(\mathbf{A}, \mathbf{B}, \mathbf{C}, \mathbf{D}, \mathbf{a}, \mathbf{b}) \backslash \mathbf{X}^{W O P}(\mathbf{c}, \mathbf{d})$, the weak optimal inverse $\operatorname{IvLP}$ problem $\mathbf{W I P}(\mathbf{c}, \mathbf{d})$ is to adjust the minimum change of $c \in \mathbf{c}, d \in \mathbf{d}$ to get new cost coefficient $(\hat{c}, \hat{d})$ such that $\bar{x} \in \mathbf{X}^{O P}(\hat{c}, \hat{d})$.

Denote by

$\mathcal{G}(\bar{x})=\left\{\hat{c} \in \mathbb{R}^{1 \times n_{1}}, \hat{d} \in \mathbb{R}^{1 \times n_{2}} \mid \min \left\{\hat{c} x^{1}+\hat{d} x^{2} \mid\left(x^{1}, x^{2}\right) \in \mathbf{X}(A, B, C, D, a, b)\right\}=\hat{c} \bar{x}^{1}+\hat{d} \bar{x}^{2}\right\}$.

Then $\mathcal{G}(\bar{x}) \neq \phi$ since $(0,0) \in \mathcal{G}(\bar{x})$. $\mathbf{W I P}(\mathbf{c}, \mathbf{d})$ can be stated as

$$
\min \left\{\|c-\hat{c}\|_{p}+\|d-\hat{d}\|_{p} \mid(\hat{c}, \hat{d}) \in \mathcal{G}(\bar{x})\right\},
$$

where $\|\cdot\|$ means the norm, we use $l_{1}$ norm in this paper.

From the analysis above, it can be easily observed that weak optimal inverse IvLP problems proposed in this paper can be transformed to inverse LP problems.

Actually, not all the cases holds for solving the weak optimal inverse IvLP problem $\mathbf{W I P}(\mathbf{c}, \mathbf{d})$. So for a given solution $\bar{x}=\left(\bar{x}^{1}, \bar{x}^{2}\right)$, we first need to test whether $\bar{x}$ is a weak solution, and then check whether it is a weak optimal solution.

Now we introduce the result of determining a vector as a weak solution to $\mathbf{F P}(\mathbf{c}, \mathbf{d})$ in [10].

Lemma 2.1. (Corollary 2 in [10]) $A$ pair $\left(\bar{x}^{1}, \bar{x}^{2}\right), \bar{x}^{1} \in \mathbb{R}^{n_{1}}, \bar{x}^{2} \in \mathbb{R}^{n_{2}}$, is a weak solution to 
the interval linear system $\mathbf{F P}(\mathbf{c}, \mathbf{d})$ if and only if there is some $s \in\{ \pm 1\}^{n_{2}}$ such that

$$
\left\{\begin{array}{l}
\underline{A} \bar{x}^{1}+\left(B^{c}-B^{\Delta} T_{s}\right) \bar{x}^{2} \leq \bar{a} \\
-\bar{A} \bar{x}^{1}-\left(B^{c}+B^{\Delta} T_{s}\right) \bar{x}^{2} \leq-\underline{a} \\
\underline{C} \bar{x}^{1}+\left(D^{c}-D^{\Delta} T_{s}\right) \bar{x}^{2} \leq \bar{b} \\
\bar{x}^{1} \geq 0
\end{array}\right.
$$

A method to check whether a weak solution is a weak optimal solution can be found in [23].

Lemma 2.2. (Theorem 3 in [23]) Define

$$
\begin{gathered}
F=\left\{r_{i} \mid i=1, \cdots, p, \bar{x}_{r_{i}}^{1}=0\right\}, \\
\left(A^{+}, B^{+}, a^{+}\right)=T_{y^{1}}(A, B, a),\left(C^{+}, D^{+}, b^{+}\right)=T_{y^{2}}(C, D, b) .
\end{gathered}
$$

$A$ weak solution $\left(\bar{x}^{1}, \bar{x}^{2}\right), \bar{x}^{1} \in \mathbb{R}^{n_{1}}, \bar{x}^{2} \in \mathbb{R}^{n_{2}}$, is weak optimal solution to $\mathbf{F P}(\mathbf{c}, \mathbf{d})$ if and only if the system

is feasible for some $\sigma \in\{ \pm 1\}^{m_{1}}$.

$$
\left\{\begin{array}{l}
A^{+} \in T_{y^{1}} \mathbf{A}, B^{+} \in T_{y^{1}} \mathbf{B}, a^{+} \in T_{y^{1}} \mathbf{a}, \\
C^{+} \in T_{y^{2}} \mathbf{C}, D^{+} \in T_{y^{2}} \mathbf{D}, b^{+} \in T_{y^{2}} \mathbf{b}, \\
A^{+} \bar{x}^{1}+B^{+} \bar{x}^{2}=a^{+}, \\
C^{+} \bar{x}^{1}+D^{+} \bar{x}^{2}=b^{+}, \\
e^{T} B^{+}+e^{T} D^{+} \in \mathbf{d}, \\
\left(e^{T} A^{+}+e^{T} C^{+}\right)_{r_{i}} \in \mathbf{c}_{r_{i}}, i=p+1, \cdots, n_{1} \\
\left(e^{T} A^{+}+e^{T} C^{+}\right)_{r_{i}} \leq \bar{c}_{r_{i}}, i=1, \cdots, p \\
y^{2} \leq 0 \\
T_{\sigma} y^{1} \geq 0
\end{array}\right.
$$

Lemma 2.3. ([22]) The solution set to $\mathbf{A} x=\mathbf{b}$ is described by

$$
\left|A^{c} x-b^{c}\right| \leq A^{\Delta}|x|+b^{\Delta} .
$$

Lemma 2.4. ([7]) An interval system $\mathbf{A} x \leq \mathbf{b}$ is weakly feasible if and only if the system

$$
A^{c} x-A^{\Delta}|x| \leq \bar{b}
$$

is feasible.

Theorem 2.1. Let $\bar{x}=\left(\bar{x}^{1}, \bar{x}^{2}\right)$ be a weak solution to the interval linear system

$$
\mathbf{A} x^{1}+\mathbf{B} x^{2}=\mathbf{a}, \mathbf{C} x^{1}+\mathbf{D} x^{2} \leq \mathbf{b}, x^{1} \geq 0 .
$$

Then $\bar{x}$ solves

$$
\tilde{A} x^{1}+\tilde{B} x^{2}=\tilde{a}, \tilde{C} x^{1}+\tilde{D} x^{2} \leq \tilde{b}, x^{1} \geq 0
$$

with

$$
\tilde{A}=A^{c}-T_{u} A^{\Delta}, \quad \tilde{B}=B^{c}-T_{u} B^{\Delta} T_{z}, \quad \tilde{C}=\underline{C}, \quad \tilde{D}=D^{c}-D^{\Delta} T_{z}, \quad \tilde{a}=a^{c}+T_{u} a^{\Delta}, \quad \tilde{b}=\bar{b},
$$
where $z=\operatorname{sign} \bar{x}^{2}$ and

$$
u_{i}=\left\{\begin{array}{ll}
\frac{\left(A^{c} \bar{x}^{1}+B^{c} \bar{x}^{2}-a^{c}\right)_{i}}{\left(A^{\Delta} \bar{x}^{1}+B^{\Delta}\left|\bar{x}^{2}\right|+a^{\Delta}\right)_{i}} & \text { if }\left(A^{\Delta} \bar{x}^{1}+B^{\Delta}\left|\bar{x}^{2}\right|+a^{\Delta}\right)_{i}>0 \\
\alpha & \text { otherwise, } \alpha \in[-1,1]
\end{array} \quad i=1, \cdots, m_{1} .\right.
$$


Proof. As $\bar{x}$ is a weak solution to $\mathbf{A} x^{1}+\mathbf{B} x^{2}=\mathbf{a}, x^{1} \geq 0$, according to Lemma 2.3, we have

$$
\left|A^{c} \bar{x}^{1}+B^{c} \bar{x}^{2}-a^{c}\right| \leq A^{\Delta}\left|\bar{x}^{1}\right|+B^{\Delta}\left|\bar{x}^{2}\right|+a^{\Delta}=A^{\Delta} \bar{x}^{1}+B^{\Delta}\left|\bar{x}^{2}\right|+a^{\Delta} .
$$

Let

$$
u_{i}=\left\{\begin{array}{ll}
\frac{\left(A^{c} \bar{x}^{1}+B^{c} \bar{x}^{2}-a^{c}\right)_{i}}{\left(A^{\Delta} \bar{x}^{1}+B^{\Delta}\left|\bar{x}^{2}\right|+a^{\Delta}\right)_{i}} & \text { if }\left(A^{\Delta} \bar{x}^{1}+B^{\Delta}\left|\bar{x}^{2}\right|+a^{\Delta}\right)_{i}>0 \\
\alpha & \text { otherwise, } \alpha \in[-1,1]
\end{array} \quad i=1, \cdots, m_{1} .\right.
$$

By (8), $|u| \leq e$, and

$$
A^{c} \bar{x}^{1}+B^{c} \bar{x}^{2}-a^{c}=T_{u}\left(A^{\Delta} \bar{x}^{1}+B^{\Delta}\left|\bar{x}^{2}\right|+a^{\Delta}\right) .
$$

Let $z=\operatorname{sign} \bar{x}^{2}$, then $\left|\bar{x}^{2}\right|=T_{z} \bar{x}^{2}$. So

$$
\left(A^{c}-T_{u} A^{\Delta}\right) \bar{x}^{1}+\left(B^{c}-T_{u} B^{\Delta} T_{z}\right) \bar{x}^{2}=a^{c}+T_{u} a^{\Delta} .
$$

Because $|u| \leq e,|z|=e$, we know $\left|T_{u} A^{\Delta}\right| \leq A^{\Delta},\left|T_{u} B^{\Delta} T_{z}\right| \leq B^{\Delta},\left|T_{u} a^{\Delta}\right| \leq a^{\Delta}$. Let

$$
\tilde{A}=A^{c}-T_{u} A^{\Delta}, \tilde{B}=B^{c}-T_{u} B^{\Delta} T_{z}, \tilde{a}=a^{c}+T_{u} a^{\Delta},
$$

then $\tilde{A} \in \mathbf{A}, \tilde{B} \in \mathbf{B}, \tilde{a} \in \mathbf{a}$, and $\bar{x}$ solves $\tilde{A} x^{1}+\tilde{B} x^{2}=\tilde{a}, x^{1} \geq 0$.

As $\bar{x}$ is a weak solution to $\mathbf{C} x^{1}+\mathbf{D} x^{2} \leq \mathbf{b}, x^{1} \geq 0$, according to Lemma 2.4, we have

$$
C^{c} \bar{x}^{1}+D^{c} \bar{x}^{2}-C^{\Delta}\left|\bar{x}^{1}\right|-D^{\Delta}\left|\bar{x}^{2}\right|=C^{c} \bar{x}^{1}+D^{c} \bar{x}^{2}-C^{\Delta} \bar{x}^{1}-D^{\Delta}\left|\bar{x}^{2}\right| \leq \bar{b} .
$$

That is, $\underline{C} \bar{x}^{1}+\left(D^{c}-D^{\Delta} T_{z}\right) \bar{x}^{2} \leq \bar{b}$. Let

$$
\tilde{C}=\underline{C}, \tilde{D}=D^{c}-D^{\Delta} T_{z}, \tilde{b}=\bar{b} .
$$

Obviously, $\tilde{C} \in \mathbf{C}, \tilde{D} \in \mathbf{D}, \tilde{b} \in \mathbf{b}$, and $\bar{x}$ solves $\tilde{C} x^{1}+\tilde{D} x^{2} \leq \tilde{b}, x^{1} \geq 0$.

Hence, the proof is completed.

We next recall KKT conditions.

Every scenario of $\mathbf{F P}(\mathbf{c}, \mathbf{d})$ can be stated as the LP problem $\mathbf{F P}(c, d)$, its corresponding dual linear program is defined as

$$
\operatorname{DFP}(c, d): \max y^{1} a+y^{2} b \text { s.t. } y^{1} A+y^{2} C \leq c, y^{1} B+y^{2} D=d, y^{2} \leq 0 .
$$

KKT conditions [3] of $\mathbf{F P}(c, d)$ is presented as follows.

Theorem 2.2. A vector $\bar{x}$ is an optimal solution to $\mathbf{F P}(c, d)$ if and only if there exists row vector $y^{1} \in \mathbb{R}^{1 \times m_{1}}, y^{2} \in \mathbb{R}^{1 \times m_{2}}$ which solves

$$
\left\{\begin{array}{l}
A \bar{x}^{1}+B \bar{x}^{2}=a \\
C \bar{x}^{1}+D \bar{x}^{2} \leq b \\
\bar{x}^{1} \geq 0 \\
y^{1} A+y^{2} C \leq c \\
y^{1} B+y^{2} D=d \\
y^{2} \leq 0 \\
{\left[c-\left(y^{1} A+y^{2} C\right)\right] \bar{x}^{1}=0} \\
y^{2}\left(C \bar{x}^{1}+D \bar{x}^{2}-b\right)=0
\end{array}\right.
$$




\section{$\S 3$ Solving Weak Optimal Inverse IvLP Problems}

\subsection{An Equivalent Characterization of Weak Optimal Inverse IvLP Problems}

Now we introduce the result of the inverse problem of LP problem $\min \{c x \mid A x=b, x \geq 0\}$ in $[27]$.

Lemma 3.1. (Theorem 2.4 in [27]) Denote by

$$
\mathcal{F}(\bar{x})=\left\{\hat{c} \in \mathbb{R}^{1 \times n} \mid \min \{\hat{c} x \mid A x=b, x \geq 0\}=\hat{c} \bar{x}\right\} .
$$

The inverse LP problem

$$
\min \left\{\|c-\hat{c}\|_{1} \mid \hat{c} \in \mathcal{F}(\bar{x})\right\}
$$

is equivalent to

$$
\begin{array}{ll}
\min & \|\theta\|_{1} \\
\text { s.t. } & y A_{., k}-\theta_{k}=c_{k}, k \in \bar{K}, \\
& y A_{., k}-\theta_{k} \leq c_{k}, k \in K, \\
& \theta_{k} \geq 0, k \in K,
\end{array}
$$

where $K=\left\{k \mid \bar{x}_{k}=0\right\}, \bar{K}=\left\{k \mid \bar{x}_{k}>0\right\}$.

In order to find the equivalent form like Lemma 3.1 for the weak optimal inverse IvLP problem $\mathbf{W I P}(\mathbf{c}, \mathbf{d})$, the following lemma is first derived.

Lemma 3.2. Let $\bar{x} \in \mathbf{X}(A, B, C, D, a, b)$. Define

$$
\begin{gathered}
F=\left\{r_{i} \mid i=1, \cdots, p, \bar{x}_{r_{i}}^{1}=0\right\} \\
G=\left\{t_{j} \mid j=1, \cdots, q, C_{t_{j}, .} \bar{x}^{1}+D_{t_{j},} \bar{x}^{2}=b_{t_{j}}\right\} .
\end{gathered}
$$

Then $\bar{x}$ is an optimal solution to $\mathbf{F P}(c, d)$ if and only if there exists $y$ such that

$$
\left\{\begin{array}{l}
y^{1} A_{., r_{i}}+y^{2} C_{., r_{i}} \leq c_{r_{i}}, i=1, \ldots, p \\
y^{1} A_{., r_{i}}+y^{2} C_{., r_{i}}=c_{r_{i}}, i=p+1, \ldots, n_{1}, \\
y^{1} B+y^{2} D=d \\
y_{t_{j}}^{2} \leq 0, j=1, \ldots, q \\
y_{t_{j}}^{2}=0, j=q+1, \ldots, m_{2} .
\end{array}\right.
$$

Proof. "Only if": If $\bar{x}$ is an optimal solution to $\mathbf{F P}(c, d)$, according to Theorem 2.2 , then there exists $\bar{y}$ solves $(9 \mathrm{a}-9 \mathrm{~h})$. Thus we have

$$
\bar{y}^{1} B+\bar{y}^{2} D=d
$$

By $(9 \mathrm{~g})$ and $F$,

$$
\begin{gathered}
\bar{y}^{1} A_{., r_{i}}+\bar{y}^{2} C_{., r_{i}} \leq c_{r_{i}}, i=1, \ldots, p \\
\bar{y}^{1} A_{., r_{i}}+\bar{y}^{2} C_{., r_{i}}=c_{r_{i}}, i=p+1, \ldots, n_{1} .
\end{gathered}
$$


By (9h) and $G$, we have

$$
\begin{gathered}
\bar{y}_{t_{j}}^{2} \leq 0, j=1, \ldots, q, \\
\bar{y}_{t_{j}}^{2}=0, j=q+1, \ldots, m_{2} .
\end{gathered}
$$

Therefore, $\bar{y}$ solves (10a-10e).

"If": As $\bar{x} \in \mathbf{X}(A, B, C, D, a, b),(9 a-9 c)$ apparently holds. If there exists $\bar{y}$ solves (10a-10e), then we have

$$
\left\{\begin{array}{l}
\bar{y}^{1} A+\bar{y}^{2} C \leq c, \\
\bar{y}^{1} B+\bar{y}^{2} D=d, \\
\bar{y}^{2} \leq 0 .
\end{array}\right.
$$

By (10a),(10b) and $F$, we obtain that

$$
\left[c_{r_{i}}-\left(\bar{y}^{1} A_{., r_{i}}+\bar{y}^{2} C_{., r_{i}}\right)\right] \bar{x}^{1}=\left\{\begin{array}{l}
{\left[c_{r_{i}}-\left(\bar{y}^{1} A_{., r_{i}}+\bar{y}^{2} C_{., r_{i}}\right)\right] \cdot 0=0, i=1, \ldots, p,} \\
0 \cdot \bar{x}^{1}=0, i=p+1, \ldots, n_{1} .
\end{array}\right.
$$

Therefore,

$$
\left[c-\left(\bar{y}^{1} A+\bar{y}^{2} C\right)\right] \bar{x}^{1}=0 .
$$

By $(10 \mathrm{~d}),(10 \mathrm{e})$ and $G$, we obtain that

$$
\bar{y}_{t_{j}}^{2}\left(C_{t_{j},}, \bar{x}^{1}+D_{t_{j},}, \bar{x}^{2}-b_{t_{j}}\right)=\left\{\begin{array}{l}
\bar{y}_{t_{j}}^{2} \cdot 0=0, j=1, \ldots, q, \\
0 \cdot\left(C_{t_{j},}, \bar{x}^{1}+D_{t_{j},}, \bar{x}^{2}-b_{t_{j}}\right)=0, j=q+1, \ldots, m_{2} .
\end{array}\right.
$$

Thus,

$$
\bar{y}^{2}\left(C \bar{x}^{1}+D \bar{x}^{2}-b\right)=0 .
$$

Hence, there exists $y$ satisfying $(9 \mathrm{a}-9 \mathrm{~h})$. According to Theorem $2.2, \bar{x}$ is an optimal solution to $\mathbf{F P}(c, d)$.

Lemma 3.3. Let

Define

$$
\begin{gathered}
F=\left\{r_{i} \mid i=1, \cdots, p, \bar{x}_{r_{i}}^{1}=0\right\}, \\
G=\left\{t_{j} \mid j=1, \cdots, q, C_{t_{j},}, \bar{x}^{1}+D_{t_{j},}, \bar{x}^{2}=b_{t_{j}}\right\} .
\end{gathered}
$$

$$
\begin{aligned}
& \mathcal{G}(\bar{x})=\{\hat{c}\left.\in \mathbb{R}^{1 \times n_{1}}, \hat{d} \in \mathbb{R}^{1 \times n_{2}} \mid \min \left\{\hat{c} x^{1}+\hat{d} x^{2} \mid\left(x^{1}, x^{2}\right) \in \mathbf{X}(A, B, C, D, a, b)\right\}=\hat{c} \bar{x}^{1}+\hat{d} \bar{x}^{2}\right\} . \\
& \mathcal{G}^{\prime}(\bar{x}) \quad=\left\{c^{*} \in \mathbb{R}^{1 \times n_{1}}, d^{*} \in \mathbb{R}^{1 \times n_{2}} \mid \min \left\{c^{*} x^{1}+d^{*} x^{2} \mid\left(x^{1}, x^{2}\right) \in \mathbf{X}(A, B, C, D, a, b)\right\}\right. \\
&\left.=c^{*} \bar{x}^{1}+d^{*} \bar{x}^{2}\right\},
\end{aligned}
$$

where $c^{*}=c+\theta, d^{*}=d+\eta,\left(y^{1}, y^{2}, \theta, \eta\right)$ is a solution to the system

$$
\left\{\begin{array}{l}
y^{1} A_{., r_{i}}+y^{2} C_{., r_{i}}-\theta_{r_{i}} \leq c_{r_{i}}, i=1, \ldots, p, \\
y^{1} A_{., r_{i}}+y^{2} C_{., r_{i}}-\theta_{r_{i}}=c_{r_{i}}, i=p+1, \ldots, n_{1}, \\
y^{1} B+y^{2} D-\eta=d, \\
y_{t_{j}}^{2} \leq 0, j=1, \ldots, q, \\
y_{t_{j}}^{2}=0, j=q+1, \ldots, m_{2}, \\
\theta_{r_{i}} \geq 0, i=1, \ldots, p .
\end{array}\right.
$$




\section{Then}

$$
\min \left\{\left\|c-c^{*}\right\|_{1}+\left\|d-d^{*}\right\|_{1} \mid\left(c^{*}, d^{*}\right) \in \mathcal{G}^{\prime}(\bar{x})\right\}=\min \left\{\|c-\hat{c}\|_{1}+\|d-\hat{d}\|_{1} \mid(\hat{c}, \hat{d}) \in \mathcal{G}(\bar{x})\right\} .
$$

Proof. For any $\left(c^{*}, d^{*}\right) \in \mathcal{G}^{\prime}(\bar{x})$, as $\left(y^{1}, y^{2}, \theta, \eta\right)$ is the solution to the system (14), and $c^{*}=c+\theta, d^{*}=d+\eta$, we have

$$
\left\{\begin{array}{l}
y^{1} A_{., r_{i}}+y^{2} C_{., r_{i}} \leq c_{r_{i}}^{*}, i=1, \ldots, p \\
y^{1} A_{., r_{i}}+y^{2} C_{., r_{i}}=c_{r_{i}}^{*}, i=p+1, \ldots, n_{1}, \\
y^{1} B+y^{2} D=d^{*} \\
y_{t_{j}}^{2} \leq 0, j=1, \ldots, q \\
y_{t_{j}}^{2}=0, j=q+1, \ldots, m_{2} .
\end{array}\right.
$$

From Lemma 3.2 , we know $\left(c^{*}, d^{*}\right) \in \mathcal{G}(\bar{x})$. So $\mathcal{G}^{\prime}(\bar{x}) \subseteq \mathcal{G}(\bar{x})$. Thus,

$\min \left\{\left\|c-c^{*}\right\|_{1}+\left\|d-d^{*}\right\|_{1} \mid\left(c^{*}, d^{*}\right) \in \mathcal{G}^{\prime}(\bar{x})\right\} \geq \min \left\{\|c-\hat{c}\|_{1}+\|d-\hat{d}\|_{1} \mid(\hat{c}, \hat{d}) \in \mathcal{G}(\bar{x})\right\}$.

By Lemma 3.2 , for any $(\hat{c}, \hat{d}) \in \mathcal{G}(\bar{x})$, there exist $\left(\bar{y}^{1}, \bar{y}^{2}\right), \bar{y}^{1} \in \mathbb{R}^{1 \times m_{1}}, \bar{y}^{2} \in \mathbb{R}^{1 \times m_{2}}$ such that

$$
\left\{\begin{array}{l}
\bar{y}^{1} A_{., r_{i}}+\bar{y}^{2} C_{., r_{i}} \leq \hat{c}_{r_{i}}, i=1, \ldots, p, \\
\bar{y}^{1} A_{., r_{i}}+\bar{y}^{2} C_{., r_{i}}=\hat{c}_{r_{i}}, i=p+1, \ldots, n_{1}, \\
\bar{y}^{1} B+\bar{y}^{2} D=\hat{d} \\
\bar{y}_{t_{j}}^{2} \leq 0, j=1, \ldots, q, \\
\bar{y}_{t_{j}}^{2}=0, j=q+1, \ldots, m_{2} .
\end{array}\right.
$$

If $\hat{c}_{r_{i}} \geq c_{r_{i}}, i=1, \ldots, p$, put $c^{*}=\hat{c}=c+\theta, d^{*}=\hat{d}=d+\eta$, then $\theta_{r_{i}} \geq 0, i=1, \ldots, p$. And (16) can be reduced to

$$
\left\{\begin{array}{l}
\bar{y}^{1} A_{., r_{i}}+\bar{y}^{2} C_{., r_{i}} \leq c_{r_{i}}+\theta_{r_{i}}, i=1, \ldots, p, \\
\bar{y}^{1} A_{., r_{i}}+\bar{y}^{2} C_{., r_{i}}=c_{r_{i}}+\theta_{r_{i}}, i=p+1, \ldots, n_{1}, \\
\bar{y}^{1} B+\bar{y}^{2} D=d+\eta \\
\bar{y}_{t_{j}}^{2} \leq 0, j=1, \ldots, q, \\
\bar{y}_{t_{j}}^{2}=0, j=q+1, \ldots, m_{2} .
\end{array}\right.
$$

So we know $\left(\bar{y}^{1}, \bar{y}^{2}, \theta, \eta\right)$ is a solution to $(14)$. Thus $(\hat{c}, \hat{d}) \in \mathcal{G}^{\prime}(\bar{x}),\left(c^{*}, d^{*}\right) \in \mathcal{G}^{\prime}(\bar{x})$ and

$$
\left\|c-c^{*}\right\|_{1}+\left\|d-d^{*}\right\|_{1}=\|c-\hat{c}\|_{1}+\|d-\hat{d}\|_{1} .
$$

If $\hat{c}_{r_{i}}<c_{r_{i}}, i=1, \ldots, p$, let

$$
\begin{aligned}
& c_{r_{i}}^{*}=\left\{\begin{array}{l}
c_{r_{i}}=c_{r_{i}}+\theta_{r_{i}}, i=1, \ldots, p, \\
\hat{c}_{r_{i}}=c_{r_{i}}+\theta_{r_{i}}, i=p+1, \ldots, n_{1},
\end{array}\right. \\
& d^{*}=\hat{d}=d+\eta .
\end{aligned}
$$

Then $\theta_{r_{i}}=0, i=1, \ldots, p$. Hence we have

$$
\left\{\begin{array}{l}
\bar{y}^{1} A_{., r_{i}}+\bar{y}^{2} C_{., r_{i}} \leq \hat{c}_{r_{i}}<c_{r_{i}}+\theta_{r_{i}}, i=1, \ldots, p, \\
\bar{y}^{1} A_{., r_{i}}+\bar{y}^{2} C_{., r_{i}}=\hat{c}_{r_{i}}=c_{r_{i}}+\theta_{r_{i}}, i=p+1, \ldots, n_{1}, \\
\bar{y}^{1} B+\bar{y}^{2} D=\hat{d}=d^{*} \\
\bar{y}_{t_{j}}^{2} \leq 0, j=1, \ldots, q, \\
\bar{y}_{t_{j}}^{2}=0, j=q+1, \ldots, m_{2} .
\end{array}\right.
$$


So we know $\left(\bar{y}^{1}, \bar{y}^{2}, \theta, \eta\right)$ is a solution to $(14)$. Thus $\left(c^{*}, d^{*}\right) \in \mathcal{G}^{\prime}(\bar{x})$ and

$$
\left|c_{r_{i}}-c_{r_{i}}^{*}\right|= \begin{cases}0 & i=1, \ldots, p, \\ \left|c_{r_{i}}-\hat{c}_{r_{i}}\right| & i=p+1, \ldots, n_{1} .\end{cases}
$$

That is $\left|c-c^{*}\right| \leq|c-\hat{c}|$ and it follows that

$$
\left\|c-c^{*}\right\|_{1}+\left\|d-d^{*}\right\|_{1} \leq\|c-\hat{c}\|_{1}+\|d-\hat{d}\|_{1} .
$$

Thus for any $(\hat{c}, \hat{d}) \in \mathcal{G}(\bar{x})$, there exists $\left(c^{*}, d^{*}\right) \in \mathcal{G}^{\prime}(\bar{x})$ such that (19) holds.

So

$\min \left\{\left\|c-c^{*}\right\|_{1}+\left\|d-d^{*}\right\|_{1} \mid\left(c^{*}, d^{*}\right) \in \mathcal{G}^{\prime}(\bar{x})\right\} \leq \min \left\{\|c-\hat{c}\|_{1}+\|d-\hat{d}\|_{1} \mid(\hat{c}, \hat{d}) \in \mathcal{G}(\bar{x})\right\}$.

Therefore,

$\min \left\{\left\|c-c^{*}\right\|_{1}+\left\|d-d^{*}\right\|_{1} \mid\left(c^{*}, d^{*}\right) \in \mathcal{G}^{\prime}(\bar{x})\right\}=\min \left\{\|c-\hat{c}\|_{1}+\|d-\hat{d}\|_{1} \mid(\hat{c}, \hat{d}) \in \mathcal{G}(\bar{x})\right\}$.

Note that in (14), the adjustment of $\theta_{r_{i}}<0, i=1, \ldots, p$ is unnecessary.

As $\theta_{r_{i}}=0, i=1, \ldots, p$ has met the optimality, and the less adjustment, the better, so we just need set $\theta_{r_{i}} \geq 0, i=1, \ldots, p$.

In combination with Lemma 3.1, Lemma 3.2 and Lemma 3.3, we have the following result.

\section{Theorem 3.1. Define}

$$
\mathcal{M}(\bar{x})=\{A \in \mathbf{A}, B \in \mathbf{B}, C \in \mathbf{C}, D \in \mathbf{D}, a \in \mathbf{a}, b \in \mathbf{b} \mid \bar{x} \in \mathbf{X}(A, B, C, D, a, b)\} .
$$

The weak optimal inverse IvLP problem $\mathbf{W I P}(\mathbf{c}, \mathbf{d})$ is equivalent to the optimization problem

$$
\begin{array}{ll}
\min & \|\theta\|_{1}+\|\eta\|_{1} \\
\text { s.t. } & y^{1} A_{., r_{i}}+y^{2} C_{., r_{i}}-\theta_{r_{i}} \leq c_{r_{i}}, i=1, \ldots, p, \\
& y^{1} A_{., r_{i}}+y^{2} C_{., r_{i}}-\theta_{r_{i}}=c_{r_{i}}, i=p+1, \ldots, n_{1}, \\
& y^{1} B+y^{2} D-\eta=d, \\
& y_{t_{j}}^{2} \leq 0, j=1, \ldots, q, \\
& y_{t_{j}}^{2}=0, j=q+1, \ldots, m_{2}, \\
& \theta_{r_{i}} \geq 0, i=1, \ldots, p,
\end{array}
$$

for any $(A, B, C, D, a, b) \in \mathcal{M}(\bar{x})$ and any $c \in \mathbf{c}, d \in \mathbf{d}$.

\subsection{The Simplification of Weak Optimal Inverse IvLP Problems}

Let

$$
\begin{gathered}
F=\left\{r_{i} \mid i=1, \cdots, p, \bar{x}_{r_{i}}^{1}=0\right\}, \\
H=\left\{s_{h} \mid h=1, \cdots, k, \bar{x}_{s_{h}}^{2}=0\right\} .
\end{gathered}
$$

For variables $\bar{x}_{r_{i}}^{1}=0, i=1, \ldots, p, \bar{x}_{s_{h}}^{2}=0, h=1, \ldots, k$, no matter how much their cost coefficients are adjusted,

$$
\left(c_{r_{i}}+\theta_{r_{i}}\right) \bar{x}_{r_{i}}^{1}=0, i=1, \ldots, p
$$




$$
\left(d_{s_{h}}+\eta_{s_{h}}\right) \bar{x}_{s_{h}}^{2}=0, h=1, \ldots, k .
$$

That is, they have no effect on the objective function value. Because the weak optimal inverse IvLP problem $\mathbf{W I P}(\mathbf{c}, \mathbf{d})$ is to adjust the minimum change of $c \in \mathbf{c}, d \in \mathbf{d}$ to obtain new cost coefficients $(\hat{c}, \hat{d})$ such that $\bar{x} \in \mathbf{X}^{O P}(\hat{c}, \hat{d})$, we had better not adjust the cost coefficient $\theta_{r_{i}}=0, i=1, \ldots, p, \eta_{s_{h}}=0, h=1, \ldots, k$.

Thus we have

$$
\begin{gathered}
\theta_{r_{i}}=0, i=1, \ldots, p, \\
\eta_{s_{h}}=0, h=1, \ldots, k .
\end{gathered}
$$

It not only simplifies the weak optimal inverse IvLP problem $\mathbf{W I P}(\mathbf{c}, \mathbf{d})$ but also significantly reduces the amount of calculation.

The objective function in $(20)$ is

$$
\min \sum_{j=1}^{n_{1}}\left|\theta_{r_{i}}\right|+\sum_{h=1}^{n_{2}}\left|\eta_{s_{h}}\right|,
$$

which can be transformed to a LP problem.

Put

$$
\begin{gathered}
\theta_{r_{i}}=\theta_{r_{i}}^{1}-\theta_{r_{i}}^{2}, \quad\left|\theta_{r_{i}}\right|=\theta_{r_{i}}^{1}+\theta_{r_{i}}^{2}, \theta_{r_{i}}^{1}, \theta_{r_{i}}^{2} \geq 0, i=p+1, \ldots, n_{1}, \\
\eta_{s_{h}}=\eta_{s_{h}}^{1}-\eta_{s_{h}}^{2}, \quad\left|\eta_{s_{h}}\right|=\eta_{s_{h}}^{1}+\eta_{s_{h}}^{2}, \eta_{s_{h}}^{1}, \eta_{s_{h}}^{2} \geq 0, h=k+1, \ldots, n_{2},
\end{gathered}
$$

then there is no absolute value in (20).

From the above discussion, we have the following result.

Theorem 3.2. The weak optimal inverse IvLP problem $\mathbf{W I P}(\mathbf{c}, \mathbf{d})$ is equivalent to the optimization problem

$$
\begin{array}{ll}
\min & \sum_{i=p+1}^{n_{1}}\left(\theta_{r_{i}}^{1}+\theta_{r_{i}}^{2}\right)+\sum_{h=k+1}^{n_{2}}\left(\eta_{s_{h}}^{1}+\eta_{s_{h}}^{2}\right) \\
\text { s.t. } & y^{1} A_{., r_{i}}+y^{2} C_{., r_{i}} \leq c_{r_{i}}, i=1, \ldots, p \\
& y^{1} A_{., r_{i}}+y^{2} C_{., r_{i}}-\theta_{r_{i}}^{1}+\theta_{r_{i}}^{2}=c_{r_{i}}, i=p+1, \ldots, n_{1}, \\
& y^{1} B_{., s_{h}}+y^{2} D_{., s_{h}}=d_{s_{h}}, h=1, \ldots, k \\
& y^{1} B_{., s_{h}}+y^{2} D_{., s_{h}}-\eta_{s_{h}}^{1}+\eta_{s_{h}}^{2}=d_{s_{h}}, h=k+1, \ldots, n_{2}, \\
& y_{t_{j}}^{2} \leq 0, j=1, \ldots, q, \\
& y_{t_{j}}^{2}=0, j=q+1, \ldots, m_{2}, \\
& \theta_{r_{i}}^{1}, \theta_{r_{i}}^{2} \geq 0, i=p+1, \ldots, n_{1}, \\
& \eta_{s_{h}}^{1}, \eta_{s_{h}}^{2} \geq 0, h=k+1, \ldots, n_{2},
\end{array}
$$

for any $(A, B, C, D, a, b) \in \mathcal{M}(\bar{x})$ and any $c \in \mathbf{c}, d \in \mathbf{d}$.

Then we can get the new cost coefficient $(\hat{c}, \hat{d})$ as

$$
\begin{aligned}
& \hat{c}=c+\theta, \\
& \hat{d}=d+\eta,
\end{aligned}
$$


where

$$
\begin{gathered}
\theta_{r_{i}}=0, i=1, \ldots, p, \\
\theta_{r_{i}}=\theta_{r_{i}}^{1}-\theta_{r_{i}}^{2}, i=p+1, \ldots, n_{1}, \\
\eta_{s_{h}}=0, i=1, \ldots, p, \\
\eta_{s_{h}}=\eta_{s_{h}}^{1}-\eta_{s_{h}}^{2}, h=k+1, \ldots, n_{2} .
\end{gathered}
$$

Algorithm. The complete description of the algorithm for solving the weak optimal inverse IvLP problem $\mathbf{W I P}(\mathbf{c}, \mathbf{d})$ can be stated as follows.

Step 1: Input $\bar{x}$, verify whether there is some $s \in\{ \pm 1\}^{n_{2}}$ such that (4) is feasible.

If not, $\bar{x}$ is not weak solution to $\mathbf{F P}(\mathbf{c}, \mathbf{d})$, end;

else, next.

Step 2: Test whether there is some $\sigma \in\{ \pm 1\}^{m_{1}}$ such that (5) is feasible.

If it is, $\bar{x}$ is weak optimal solution to $\mathbf{F P}(\mathbf{c}, \mathbf{d})$, end;

else, next.

Step 3: Construct $(A, B, C, D, a, b) \in \mathcal{M}(\bar{x})$.

Step 4: Traverse $c, d$ in $[\underline{c}, \bar{c}],[\underline{d}, \bar{d}]$ to solve $(21)$.

Step 5: Return $\left(y^{1}, y^{2}, \theta_{1}, \eta\right)$, optimal value, and the new cost coefficient $(\hat{c}, \hat{d})$.

Remark. In step 1 and step 2, there are $2^{n_{2}}$ and $2^{m_{1}}$ linear systems respectively. For step 1 , we can continue as soon as we find some $s \in\{ \pm 1\}^{n_{2}}$ such that (4) is feasible. However, for step 2 , we can not continue until all the systems have been judged. For step 3 , we put $\bar{x}$ into the constraint system of $\mathbf{F P}(\mathbf{c}, \mathbf{d})$, as $\bar{x}$ is given, it can be transformed into a system of equations and inequalities about $A, B, C, D, a, b$, which can be solvable by software. Usually, there are an infinite number of solutions, which can be assigned according to actual requirements. They can also be constructed according to some special conclusions, such as the case in Theorem 2.1, for the fact that $(\tilde{A}, \tilde{B}, \tilde{C}, \tilde{D}, \tilde{a}, \tilde{b}) \in \mathcal{M}(\bar{x})$.

\section{$\S 4$ Conclusion}

In this paper, we introduced weak optimal inverse IvLP problems. An equivalent characterization of weak optimal inverse IvLP problems was given, and the problem was simplified without adjusting the cost coefficient of null variable. Future works will include inverse IvLP problems when the feasible region also needs to be changed and problems in other formulations, for example, strong optimal inverse IvLP problems.

Open Access This article is licensed under a Creative Commons Attribution 4.0 International License, which permits use, sharing, adaptation, distribution and reproduction in any medium or format, as long as you give appropriate credit to the original author(s) and the source, provide a link to the Creative Commons licence, and indicate if changes were made. The images or other third party material in this article are included in the articles Creative Commons 
licence, unless indicated otherwise in a credit line to the material. If material is not included in the articles Creative Commons licence and your intended use is not permitted by statutory regulation or exceeds the permitted use, you will need to obtain permission directly from the copyright holder. To view a copy of this licence, visit http://creativecommons.org/licenses/by/4.0/.

\section{References}

[1] S Ahmed, Y Guan. The inverse optimal value problem, Mathematical Programming, 2005, 102(1): 91-110.

[2] R K Ahuja, J B Orlin. Inverse optimization, Operations Research, 2001, 49(5): 771-783.

[3] M S Bazaraa, J J Jarvis. Linear programming and network flows, J Wiley, New York, 1977.

[4] D Burton, P L Toint. On the use of an inverse shortest paths algorithm for recovering linearly correlated costs, Mathematical Programming, 1994, 63(1): 1-22.

[5] M Fiedler, J Nedoma, J Ramík, J Rohn, K Zimmermann. Linear optimization problems within exact data, Springer-Verlag, New York, 2006.

[6] C Finn, S Levine, P Abbeel. Guided cost learning: Deep inverse optimal control via policy optimization, JMLR: W\&CP, 2016, 48: 49-58.

[7] W Gerlach. Zur lösung linearer ungleichungssysteme bei störimg der rechten seite und der koeffizientenmatrix, Mathematische Operationsforschung und Statistik, Series Optimization, 1981, 12(1): 41-43.

[8] X Guan, P M Pardalos, B Zhang. Inverse max+sum spanning tree problem under weighted $l_{1}$ norm by modifying the sum-cost vector, Optimization Letters, 2018, 12(5): 1065-1077.

[9] M Hladík. Interval linear programming: A survey, Chapter 2, In: Mann ZA (ed) Linear Programming New Frontiers in Theory and Applications, Nova Science, New York, 2012, 85-120.

[10] M Hladík. Weak and strong solvability of interval linear systems of equations and inequalities, Linear Algebra and Its Applications, 2013, 438(11): 4156-4165.

[11] D S Hochbaum. Efficient Algorithms for the Inverse Spanning-Tree Problem, Operations Research, 2003, 51(5): 785-797.

[12] H Ishibuchi, H Tanaka. Multiobjective programming in optimization of the interval objective function, European Journal of Operational Research, 1990, 48(2): 219-225.

[13] C Jansson, S M Rump. Rigorous solution of linear programming problems with uncertain data, Zeitschrift für Operations Research, 1991, 35(2): 87-111.

[14] D Li, Y Leung, W Wu. Multiobjective interval linear programming in admissible-order vector space, Information Sciences, 2019, 486: 1-19.

[15] W Li, X Liu, H Li. Generalized solutions to interval linear programmes and related necessary and sufficient optimality conditions, Optimization Methods and Software, 2015, 30(3): 516-530. 
[16] W Li, J Luo, C Deng. Necessary and sufficient conditions of some strong optimal solutions to the interval linear programming, Linear Algebra and Its Applications, 2013, 439(10): 3241-3255.

[17] X Liu, W Li, P Liu. Construction method of constraint matrices corresponded by an optimal solution, Applied Mechanics and Materials, 2014, 513-517: 1617-1620.

[18] J Luo, W Li. Strong optimal solutions of interval linear programming, Linear Algebra and Its Applications, 2013, 439(8): 2479-2493.

[19] A Mostafaee, M Hladík, M Černỳ. Inverse linear programming with interval coefficients, Journal of Computational and Applied Mathematics, 2016, 292: 591-608.

[20] M Mohammadi, M Gentili. Bounds on the worst optimal value in interval linear programming, Soft Computing, 2019, 23(21): 11055-11061.

[21] J Novotná, M Hladík, T Masařík. Duality gap in interval linear programming, Journal of Optimization Theory and Applications, 2020, 184(2): 565-580.

[22] W Oettli, W Prager. Compatibility of approximate solution of linear equations with given error bounds for coefficients and right hand sides, Numerische Mathematik, 1964, 6(1): 405-409.

[23] M Rada, M Hladík, E Garajová. Testing weak optimality of a given solution in interval linear programming revisited: NP-hardness proof, algorithm and some polynomially-solvable cases, Optimization Letters, 2019, 13(4): 875-890.

[24] J Rohn. Strong solvability of interval linear programming problems, Computing, 1981, 26(1): 79-82.

[25] R E Steuer. Algorithms for linear programming problems with interval objective function coefficients, Mathematics of Operations Research, 1981, 6(3): 333-348.

[26] H Zhang, T Feng, G Yang. Distributed cooperative optimal control for multiagent systems on directed graphs: An inverse optimal approach, IEEE Transactions on Cybernetics, 2015, 45(7): 1315-1326.

[27] J Zhang, Z Liu. Calculating some inverse linear programming problems, Journal of Computational and Applied Mathematics, 1996, 72(2): 261-273.

${ }^{1}$ School of Statistics and Mathematics, Zhejiang Gongshang University, Hangzhou 310018, China.

${ }^{2}$ Hangzhou College of Commerce, Zhejiang Gongshang University, Hangzhou 310018, China.

Email: jtao@263.net

${ }^{3}$ School of Data Sciences, Zhejiang University of Finance and Economics, Hangzhou 310018, China. 